\title{
Correction to: The Probabilistic Model and Forecasting of Power Load Based on Variational Bayesian Expectation Maximization and Relevance Vector Machine
}

\author{
Wengen $\mathrm{Gao}^{1,2} \cdot$ Qigong $\mathrm{Chen}^{2} \cdot$ Yuan $\mathrm{Ge}^{2} \cdot$ YiQing Huang $^{2}$
}

Published online: 22 November 2018

(c) Springer Science+Business Media, LLC, part of Springer Nature 2018

\section{Correction to: Wireless Pers Commun (2018) 102:3041-3053 https://doi.org/10.1007/s11277-018-5324-2}

There was a spelling error in the first author's name in the original publication. The name is correctly shown here.

The original article can be found online at https://doi.org/10.1007/s11277-018-5324-2.

Wengen Gao

gwg69@163.com; gaowengen_ahpu@163.com

Qigong Chen

Prof_ChenQigong@163.com

Yuan Ge

ygetoby@mail.ustc.edu.cn

YiQing Huang

yiqhuang@ahpu.edu.cn

1 School of Internet of Things Engineering, Jiangnan University, Wuxi 214122, Jiangsu, China

2 AnHui Polytechnic University, WuHu 241000, AnHui, China 